This postprint is published in:

Journal of mathematical analysis and applications, Volume 486, Issue 2, June 2020, art. 123898 [18 str.]

DOI: $10.1016 /$ j.jmaa.2020.123898

\title{
EXISTENCE OF SOLUTIONS FOR SYSTEMS ARISING IN ELECTROMAGNETISM
}

\author{
M.K. HAMDANI AND D.D. REPOVŠ
}

\begin{abstract}
In this paper, we study the following $p(x)$-curl systems:
$\left\{\nabla \times\left(|\nabla \times \mathbf{u}|^{p(x)-2} \nabla \times \mathbf{u}\right)+a(x)|\mathbf{u}|^{p(x)-2} \mathbf{u}=\lambda f(x, \mathbf{u})+\mu g(x, \mathbf{u}), \quad \nabla \cdot \mathbf{u}=0, \quad\right.$ in $\Omega$, $\left\{|\nabla \times \mathbf{u}|^{p(x)-2} \nabla \times \mathbf{u} \times \mathbf{n}=0, \quad \mathbf{u} \cdot \mathbf{n}=0\right.$, on $\partial \Omega$,

where $\Omega \subset \mathbb{R}^{3}$ is a bounded simply connected domain with a $C^{1,1}$-boundary, denoted by $\partial \Omega$, $p: \bar{\Omega} \rightarrow(1,+\infty)$ is a continuous function, $a \in L^{\infty}(\Omega), f, g: \Omega \times \mathbb{R}^{3} \rightarrow \mathbb{R}^{3}$ are Carathéodory functions, and $\lambda, \mu$ are two parameters. Using variational arguments based on Fountain theorem and Dual Fountain theorem, we establish some existence and non-existence results for solutions of this problem. Our main results generalize the results of Xiang et al. (J. Math. Anal. Appl., 2017), Bahrouni and Repovš (Complex Var. Elliptic Equ., 2018), and Ge and Lu (Mediterr. J. Math., 2019).
\end{abstract}

\section{INTRODUCTION}

Since the variable exponent spaces have been thoroughly studied by Kováčik-Rákosník [30, they have been used in previous decades to model various phenomena. In the studies of a class of nonstandard variational problems and PDEs, variable exponent spaces play an important role, e.g. in electrorheological fluids [34, 35, 38, thermorheological fluids 17, 8, and image processing [5, 16, 31. For nonlinear problems with variable growth, there has been a great deal of interest in studying the existence, multiplicity, uniqueness and regularity of solutions - for the main results (as well as definitions of some these properties) see [1, 2, 3, 11, 12, 13, 14, 15, 19, 21, 22, 23, 30, 32, 33, 36, 37. and the references therein.

The $p(x)$-curl operator defined by $\nabla \times\left(|\nabla \times \mathbf{u}|^{p(x)-2} \nabla \times \mathbf{u}\right)$ is a generalization of the $p$-curl operator in which the constant exponent $p$ has been replaced by a variable exponent $p(x)$. The $p(x)$-curl systems possess more complicated structure than the $p$-curl operators, due to the fact that they are not homogeneous. Therefore the study of various mathematical problems with variable exponent is very interesting and raises many difficult mathematical problems.

Moreover, the study of nonlinear elliptic equations involving quasilinear homogeneous type operators like the $p$-Laplace or $p$-curl operators is based on the theory of standard Sobolev spaces $W^{1, p}(\Omega)$ in order to find weak solutions - see 4, 25, 27, 29. These spaces consist of functions that have weak derivatives and satisfy certain integrability conditions. In the case of nonhomogeneous $p(x)$-Laplace operators, the natural setting for this approach is to use of the variable exponent Sobolev spaces. The basic idea is to replace the Lebesgue spaces $L^{p}(\Omega)$ by more general spaces $L^{p(\cdot)}(\Omega)$, called the variable exponent Lebesgue spaces. However, in literature the only results involving the $p(x)$-curl systems by variational methods can be found in [5, 9, 10, 39.

Throughout the paper, vector functions and spaces of vector functions will be in boldface. We shall use $\partial_{x}$ to denote the partial derivative of a function with respect to the variable $x$.

Key words and phrases. Variable exponent; $p(x)$-curl system; Palais-Smale compactness condition; Dual Fountain theorem; Multiplicity of solutions; Electromagnetism.

Mathematics Subject Classification (2010): Primary: 35J55, 35J65; Secondary: 35B65. 
To introduce our problem precisely, we first give some notations. Let $\mathbf{u}=\left(u_{1}, u_{2}, u_{3}\right)$ be a vector function on $\Omega$. The divergence of $\mathbf{u}$ is denoted by

$$
\nabla \cdot \mathbf{u}=\partial_{x_{1}} u_{1}+\partial_{x_{2}} u_{2}+\partial_{x_{3}} u_{3}
$$

and the curl of $\mathbf{u}$, written curl $\mathbf{u}$ or $\nabla \times \mathbf{u}$, is defined to be the vector field

$$
\nabla \times \mathbf{u}=\left\langle\partial_{x_{2}} u_{3}-\partial_{x_{3}} u_{2}, \partial_{x_{3}} u_{1}-\partial_{x_{1}} u_{3}, \partial_{x_{1}} u_{2}-\partial_{x_{2}} u_{1}\right\rangle .
$$

Throughout this paper, unless otherwise stated, we shall always assume that exponent $p(x)$ is continuous on $\bar{\Omega}$ with

$$
1<p^{-}=\min _{x \in \bar{\Omega}} p(x) \leqslant p^{+}=\max _{x \in \bar{\Omega}} p(x)<3,
$$

and satisfies the logarithmic continuity, i.e. that there exists a function $\omega: \mathbb{R}_{0}^{+} \rightarrow \mathbb{R}_{0}^{+}$such that

(1.1for all $x, y \in \bar{\Omega},|x-y|<1,|p(x)-p(y)| \leqslant \omega(|x-y|)$, and $\lim _{\tau \rightarrow 0^{+}} \omega(\tau) \log \frac{1}{\tau}=C<\infty$.

In 2016, Antontsev-Miranda-Santos [6] studied the qualitative properties of solutions for the following $p(x, t)$-curl systems:

$$
\left\{\begin{array}{l}
\partial_{t} \mathbf{u}+\nabla \times\left(|\nabla \times \mathbf{u}|^{p(x, t)-2} \nabla \times \mathbf{u}\right)=f(\mathbf{u}), \quad \nabla \cdot \mathbf{u}=0, \text { in } \Omega \times(0, T), \\
|\nabla \times \mathbf{u}|^{p(x, t)-2} \nabla \times \mathbf{u} \times \mathbf{n}=0, \quad \mathbf{u} \cdot \mathbf{n}=0, \text { on } \partial \Omega \times(0, T) \\
\mathbf{u}(x, 0)=\mathbf{u}_{0}(x), \text { in } \Omega,
\end{array}\right.
$$

where $\nabla \times\left(|\nabla \times \mathbf{u}|^{p(x, t)-2} \nabla \times \mathbf{u}\right)$ is the $p(x, t)$-curl operator

$$
f(\mathbf{u})=\lambda \mathbf{u}\left(\int_{\Omega}|\mathbf{u}|^{2} d x\right)^{\frac{\rho-2}{2}} \text { where } \lambda \in\{-1,0,1\} \text { and } \rho \text { is a positive constant. }
$$

They introduced a suitable functional framework and a convenient basis in order to apply Galerkin's method and they studied the blow-up and finite time extinction properties of solutions, depending on the values of $\lambda$ and $\rho$. In the same year, Xiang-Wang-Zhang [39] used for the first time, the variational methods for equations involving $p(x)$-curl operator of the following type:

$$
\left\{\begin{array}{l}
\nabla \times\left(|\nabla \times \mathbf{u}|^{p(x)-2} \nabla \times \mathbf{u}\right)+a(x)|\mathbf{u}|^{p(x)-2} \mathbf{u}=f(x, \mathbf{u}), \quad \nabla \cdot \mathbf{u}=0, \text { in } \Omega, \\
|\nabla \times \mathbf{u}|^{p(x)-2} \nabla \times \mathbf{u} \times \mathbf{n}=0, \quad \mathbf{u} \cdot \mathbf{n}=0, \text { on } \partial \Omega
\end{array}\right.
$$

They studied the existence and multiplicity of solutions for system $(1.3)$ with the following assumptions on $a(x)$ and $f(x, \mathbf{u})$ :

$(\mathcal{A}): a(x) \in L^{\infty}(\Omega)$ and there exist $a_{0}, a_{1}>0$ such that $a_{0} \leqslant a(x) \leqslant a_{1}$ for all $x \in \Omega$.

$\left(H_{1}\right)$ : There exists $F: \Omega \times \mathbb{R}^{3} \rightarrow \mathbb{R}$ which is differentiable with respect to $\mathbf{u} \in \mathbb{R}^{3}$ and such that

$$
f(x, \mathbf{u})=\partial_{\mathbf{u}} F(x, \mathbf{u}): \Omega \times \mathbb{R}^{3} \rightarrow \mathbb{R}^{3}
$$

is a Carathéodory function.

$\left(H_{2}\right)$ : There exist $C>0, q \in C(\bar{\Omega})$, and

$$
1<q(x)<p^{*}(x)=\frac{3 p(x)}{3-p(x)} \text { in } \bar{\Omega}
$$

such that

$$
|f(x, \mathbf{u})| \leqslant C\left(1+|\mathbf{u}|^{q(x)-1}\right), \text { for all }(x, \mathbf{u}) \in \Omega \times \mathbb{R}^{3} .
$$

$\left(H_{3}\right)$ : There exists a constant $\mu>p^{+}$such that

$$
0<\mu F(x, \mathbf{u}) \leqslant f(x, \mathbf{u}) \cdot \mathbf{u} \text { for all } x \in \Omega \text { and } \mathbf{u} \in \mathbb{R}^{3} \backslash\{0\} .
$$

$\left(H_{4}\right): \lim \sup _{\mathbf{u} \rightarrow 0} \frac{|f(x, \mathbf{u})|}{|\mathbf{u}|^{p(x)-1}}=0$ uniformly in $x \in \Omega$.

$\left(H_{5}\right): \inf _{x \in \Omega, \mathbf{u} \in \mathbb{R}^{3},|\mathbf{u}|=1} F(x, \mathbf{u})>0$.

$\left(H_{6}\right): F(x,-\mathbf{u})=F(x, \mathbf{u})$ for all $(x, \mathbf{u}) \in \Omega \times \mathbb{R}^{3}$. 
The proofs in 39] are based on Mountain Pass theorem and Symmetric Mountain Pass theorem. Under the conditions $(\mathcal{A})$ and $\left(H_{1}\right)-\left(H_{6}\right)$, the following was proved in [39].

Theorem 1.1. (see [39, Theorems 1.1 and 1.2]). Suppose that

$$
p(x)<q(x)<\frac{3 p(x)}{3-p(x)} \quad \text { for all } x \in \bar{\Omega} .
$$

Then the following holds:

(1) If a $(x)$ satisfies $(\mathcal{A})$ and $f(x, \mathbf{u})$ satisfies $\left(H_{1}\right)-\left(H_{5}\right)$, then system (1.3) has one nontrivial mountain pass solution.

(2) If a $(x)$ satisfies $(\mathcal{A})$ and $f(x, \mathbf{u})$ satisfies $\left(H_{1}\right)-\left(H_{4}\right)$ and $\left(H_{6}\right)$, then system (1.3) has infinitely many nontrivial mountain pass solutions.

In 2019, with the same method as in [39, Ge-Lu [10, gave some weaker conditions than in 39] and they proved the existence and the multiplicity of solutions for (1.3). In 2017, Bahrouni-Repovš [9] studied the following $p(x)$-curl system

$$
\left\{\begin{array}{l}
\nabla \times\left(|\nabla \times \mathbf{u}|^{p(x)-2} \nabla \times \mathbf{u}\right)=\lambda f(x, \mathbf{u})-\mu g(x, \mathbf{u}), \quad \nabla \cdot \mathbf{u}=0, \quad \text { in } \Omega, \\
|\nabla \times \mathbf{u}|^{p(x)-2} \nabla \times \mathbf{u} \times \mathbf{n}=0, \quad \mathbf{u} \cdot \mathbf{n}=0, \text { on } \partial \Omega .
\end{array}\right.
$$

Clearly, this problem is a special case of our main system when $a \equiv 0$. Bahrouni-Repovš studied the existence of solutions for system (1.4) when $f$ satisfies $\left(H_{1}\right)$, plus the following conditions:

$\left(F_{2}\right)$ : There exist $\alpha, \beta>0$ and $q \in C(\bar{\Omega})$ such that

$$
p^{+}<q(x)<p^{*}(x)=\frac{3 p(x)}{3-p(x)} \quad \text { in } \bar{\Omega}
$$

and

$$
|F(x, \mathbf{u})| \geqslant \alpha|\mathbf{u}|^{q(x)} \text { and }|f(x, \mathbf{u})| \leqslant \beta\left(1+|\mathbf{u}|^{q(x)-1}\right) \text {, for all }(x, \mathbf{u}) \in \bar{\Omega} \times \mathbb{R}^{3} .
$$

whereas for $g$ they made the following assumptions:

$\left(G_{1}\right)$ : There exist a nonnegative function $g \in L^{\infty}(\Omega)$ and $r \in C(\bar{\Omega})$ such that

$$
p^{+}<r^{-} \leqslant r(x)<q^{-} \text {and } G(x, \mathbf{u})=g(x)|\mathbf{u}|^{r(x)}, \quad \text { for all }(x, \mathbf{u}) \in \bar{\Omega} \times \mathbb{R}^{3} .
$$

$\left(G_{2}\right): G: \Omega \times \mathbb{R}^{3} \rightarrow \mathbb{R}$ is differentiable with respect to $\mathbf{u} \in \mathbb{R}^{3}$ and $g=\partial_{\mathbf{u}} G(x, \mathbf{u}): \Omega \times \mathbb{R}^{3} \rightarrow \mathbb{R}^{3}$ is a Carathéodory function.

$\left(G_{3}\right)$ : There exist $\gamma, \mu>0, L>1$ and $k, r \in C(\bar{\Omega})$ such that $1<k<p^{-}$and $1<r(x)<p^{*}(x)$,

$$
\begin{gathered}
|g(x, \mathbf{u})| \leqslant \mu\left(1+|\mathbf{u}|^{r(x)-1}\right), \quad \text { for all }(x, \mathbf{u}) \in \bar{\Omega} \times \mathbb{R}^{3}, \\
\limsup _{\mathbf{u} \rightarrow 0} \frac{G(x, \mathbf{u})}{|\mathbf{u}|^{p^{+}}}=0 \text { uniformly in } x \in \Omega,
\end{gathered}
$$

and

$$
\sup _{\mathbf{u} \in \mathbf{W}^{p(x)}(\Omega)} \int_{\Omega} G(x, \mathbf{u}) d x>0,|G(x, \mathbf{u})| \leqslant \gamma|\mathbf{u}|^{k(x)}, \text { for all } x \in \Omega \text { and }|\mathbf{u}|>L .
$$

Under the conditions $\left(H_{1}\right),\left(F_{2}\right),\left(G_{1}\right)-\left(G_{3}\right)$, the following was proved in 9 .

Theorem 1.2. (see [9, Theorems 1.1 and 1.2])

(1) Assume that hypotheses $\left(H_{1}\right),\left(F_{2}\right),\left(G_{1}\right)-\left(G_{2}\right)$ hold. Then there exist $\lambda_{1}, \mu_{1}>0$ such that, if $0<\lambda<\lambda_{1}$ and $\mu>\mu_{1}$, then system (1.4) does not have any nontrivial weak solutions.

(2) Assume that hypotheses $\left(H_{1}\right),\left(F_{2}\right),\left(G_{1}\right)-\left(G_{2}\right)$ hold. Then for each $\mu>0$, there exists $\lambda_{\mu}>0$ such that if $\lambda>\lambda_{\mu}$, then system (1.4) has at least one nontrivial weak solution.

(3) Assume that hypotheses $\left(H_{1}\right),\left(F_{2}\right),\left(G_{2}\right)-\left(G_{3}\right)$ hold. Then there exist $\lambda_{2}, \lambda_{3}, r>0$ such that, if $\lambda \in\left[\lambda_{2}, \lambda_{3}\right]$, then there exists $\mu_{2}>0$ with the following property: for each $\mu \in\left[0, \mu_{2}\right]$, system (1.4) has at least three solutions whose norms are less than $r$. 
Motivated by these results, we study in this paper the existence of solutions for the following $p(x)$-curl systems by means of Fountain theorem and Dual Fountain theorem.

$$
\left\{\begin{array}{l}
\nabla \times\left(|\nabla \times \mathbf{u}|^{p(x)-2} \nabla \times \mathbf{u}\right)+a(x)|\mathbf{u}|^{p(x)-2} \mathbf{u}=\lambda f(x, \mathbf{u})+\mu g(x, \mathbf{u}), \quad \nabla \cdot \mathbf{u}=0, \quad \text { in } \Omega, \\
|\nabla \times \mathbf{u}|^{p(x)-2} \nabla \times \mathbf{u} \times \mathbf{n}=0, \quad \mathbf{u} \cdot \mathbf{n}=0, \text { on } \partial \Omega,
\end{array}\right.
$$

where $\Omega \subset \mathbb{R}^{3}$ is a bounded simply connected domain with a $C^{1,1}$ boundary denoted by $\partial \Omega, \lambda$ and $\mu$ are parameters, $p: \bar{\Omega} \rightarrow(1,+\infty)$ is a continuous function, $a \in L^{\infty}(\Omega)$, and $f, g: \Omega \times \mathbb{R}^{3} \rightarrow \mathbb{R}^{3}$ are Carathéodory functions.

Remark 1.3. To the best of our knowledge, there are no results concerning curl systems with variable exponent based on Fountain theorem and Dual Fountain theorem. In this context, the results of our paper can be seen as a generalization of the results above, to the $p(x)$-curl systems arising in electromagnetism.

We shall impose the following condition on $a(x)$ :

$(\mathcal{A})_{1}: a(x) \in L^{\infty}(\Omega)$ such that $\inf _{x \in \Omega} a(x)=a^{-}>0$.

We shall also assume that $f(x, \mathbf{u})$ and $g(x, \mathbf{u})$ satisfy the following global conditions:

$\left(f_{1}\right)$ : Condition $\left(H_{1}\right)$ stated above.

$\left(f_{2}\right)$ : There exist $c_{1}>0$ and $q(x) \in C(\bar{\Omega})$ such that

$$
1<p^{+}<q^{-} \leqslant q(x)<p^{*}(x)=\frac{3 p(x)}{3-p(x)} \text { in } \bar{\Omega}
$$

and

$$
|f(x, \mathbf{u})| \leqslant c_{1}|\mathbf{u}|^{q(x)-1}, \text { for all }(x, \mathbf{u}) \in \bar{\Omega} \times \mathbb{R}^{3} .
$$

$\left(f_{3}\right)$ : There are constants $l>0$ and $\theta>p^{+}$such that

$$
0<\theta F(x, \mathbf{u}) \leqslant f(x, \mathbf{u}) \cdot \mathbf{u} \text { for all }|\mathbf{u}| \geqslant l \text { and } x \in \Omega .
$$

$\left(f_{4}\right)$ : Condition $\left(H_{6}\right)$ stated above.

$\left(f_{5}\right): \limsup _{\mathbf{u} \rightarrow \infty} \frac{F(x, \mathbf{u})}{|\mathbf{u}|^{p^{-}}}=0$ uniformly in $x \in \Omega$.

$\left(f_{6}\right): f(x, \mathbf{u}) \cdot \mathbf{u}>0$ for all $(x, \mathbf{u}) \in \Omega \times \mathbb{R}^{3}$.

$\left(g_{1}\right)$ : Condition $\left(G_{2}\right)$ stated above.

$\left(g_{2}\right)$ : There exist $c_{2}>0$ and $\gamma(x) \in C(\bar{\Omega})$ such that

$$
1<\gamma(x)<\gamma^{+}<p^{-}<p^{*}(x)=\frac{3 p(x)}{3-p(x)} \text { in } \bar{\Omega}
$$

and

$$
|g(x, \mathbf{u})| \leqslant c_{2}|\mathbf{u}|^{\gamma(x)-1} \text { for all }(x, \mathbf{u}) \in \bar{\Omega} \times \mathbb{R}^{3} .
$$

$\left(g_{3}\right): G(x,-\mathbf{u})=G(x, \mathbf{u})$ for all $(x, \mathbf{u}) \in \Omega \times \mathbb{R}^{3}$.

$\left(g_{4}\right): \liminf _{\mathbf{u} \rightarrow 0} \frac{G(x, \mathbf{u})}{|\mathbf{u}|^{\alpha}} \geqslant 0$ uniformly in $x \in \Omega$ with $0<\alpha<p^{-}$.

$\left(g_{5}\right): g(x, \mathbf{u}) \cdot \mathbf{u}>0$ for all $(x, \mathbf{u}) \in \Omega \times \mathbb{R}^{3}$.

The variational structure of this problem leads us to introduce the following space

$$
\mathbf{W}^{p(x)}(\Omega)=\left\{\mathbf{v} \in \mathbf{L}^{p(x)}(\Omega): \nabla \times \mathbf{v} \in \mathbf{L}^{p(x)}(\Omega), \nabla \cdot \mathbf{v}=0,\left.\mathbf{v} \cdot \mathbf{n}\right|_{\partial \Omega}=0\right\},
$$

see Section 2.1 for more details. Let us proceed with setting system 1.5 in the variational structure. A function $\mathbf{u} \in \mathbf{W}^{p(x)}(\Omega)$ is said to be a weak solution of $\sqrt{1.5}$ if

$$
\int_{\Omega}|\nabla \times \mathbf{u}|^{p(x)-2} \nabla \times \mathbf{u} \cdot \nabla \times \mathbf{v} d x+\int_{\Omega} a(x)|\mathbf{u}|^{p(x)-2} \mathbf{u} \cdot \mathbf{v} d x=\lambda \int_{\Omega} f(x, \mathbf{u}) \cdot \mathbf{v} d x+\mu \int_{\Omega} g(x, \mathbf{u}) \cdot \mathbf{v} d x,
$$

for all $\mathbf{v} \in \mathbf{W}^{p(x)}(\Omega)$. The Euler-Lagrange functional associated to system $[1.5$ is defined by

$$
I_{\lambda, \mu}=\Phi-\lambda J-\mu \Psi, \quad \lambda, \mu \in \mathbb{R},
$$


where

$$
\begin{gathered}
\Phi(\mathbf{u})=\int_{\Omega} \frac{1}{p(x)}\left(|\nabla \times \mathbf{u}(x)|^{p(x)}+a(x)|\mathbf{u}(x)|^{p(x)}\right) d x, \\
J(\mathbf{u})=\int_{\Omega} F(x, \mathbf{u}) d x, \quad \Psi(\mathbf{u})=\int_{\Omega} G(x, \mathbf{u}) d x,
\end{gathered}
$$

and

$$
F(x, \mathbf{u})=\int_{0}^{\mathbf{u}} f(x, \mathbf{s}) d s, \quad G(x, \mathbf{u})=\int_{0}^{\mathbf{u}} g(x, \mathbf{s}) d s .
$$

Now, we can state our main results as follows.

Theorem 1.4. Assume that a $(x)$ satisfies $(\mathcal{A})_{1}$, that conditions $\left(f_{1}\right)-\left(f_{4}\right),\left(g_{1}\right)-\left(g_{3}\right)$ hold, and that $p^{+}<q^{-} \leqslant q(x) \leqslant p_{2}^{*}(x), \gamma^{+}<p^{-}$. Then system (1.5) has a sequence of weak solutions $\left( \pm \mathbf{u}_{k}\right)$ in $\mathbf{W}^{p(x)}(\Omega)$ for every $\lambda>0, \mu>0$, such that $I_{\lambda, \mu}\left( \pm \mathbf{u}_{k}\right) \rightarrow+\infty$, as $k \rightarrow+\infty$.

Theorem 1.5. Assume that a $(x)$ satisfies $(\mathcal{A})_{1}$, that conditions $\left(f_{1}\right)-\left(f_{2}\right),\left(\sqrt{f_{4}}\right),\left(g_{1}\right)-\left(g_{4}\right)$ hold, and that $p^{+}<q^{-} \leqslant q(x) \leqslant p_{2}^{*}(x), \gamma^{+}<p^{-}$. Then system (1.5) has a sequence of weak solutions $\left( \pm u_{k}\right)$ in $\mathbf{W}^{p(x)}(\Omega)$ for every $\lambda>0, \mu>0$, such that $I_{\lambda, \mu}\left( \pm \mathbf{u}_{k}\right)<0$ and $I_{\lambda, \mu}\left( \pm \mathbf{u}_{k}\right) \rightarrow 0$, as $k \rightarrow+\infty$.

Theorem 1.6. Assume that a $(x)$ satisfies $(\mathcal{A})_{1}$, that conditions $\left(f_{1}\right)-\left(f_{2}\right),\left(f_{5}\right),\left(g_{1}\right)-\left(g_{2}\right),\left(g_{4}\right)$ hold, and that $p^{+}<q^{-} \leqslant q(x) \leqslant p_{2}^{*}(x), \gamma^{+}<p^{-}$. Then system (1.5) has at least one nontrivial weak solution in $\mathbf{W}^{p(x)}(\Omega)$ for every $\lambda<0, \mu>0$.

Theorem 1.7. Assume that a $(x)$ satisfies $(\mathcal{A})_{1}$. If $\left(f_{1}\right)-\left(f_{2}\right),\left[f_{6}\right), g_{1}-\left(g_{2},-g_{5}\right.$ hold and $p^{+}<$ $q^{-} \leqslant q(x) \leqslant p_{2}^{*}(x), \gamma^{+}<p^{-}$. Then system (1.5) has no nontrivial weak solution in $\mathbf{W}^{p(x)}(\Omega)$ for every $\lambda<0, \mu<0$.

We conclude with an outline of the structure of the paper. In Section 2, we introduce some preliminary results and in Section 3 , we give the proofs of the main results.

\section{Preliminaries}

In this section we shall give some preliminary results which will be used in the sequel.

2.1. Variable exponent Lebesgue and Sobolev spaces. To study our problems, we shall need to introduce certain function spaces. Denote

$$
C_{+}(\Omega)=\left\{p \in C(\bar{\Omega}): \min _{x \in \Omega} p(x)>1\right\} .
$$

Definition 2.1. The variable exponent Lebesgue space $L^{p(x)}(\Omega)$ is defined by

$$
L^{p(x)}(\Omega)=\left\{\mathbf{u} ; \mathbf{u} \text { is a measurable real-valued function such that } \int_{\Omega}|\mathbf{u}|^{p(x)} d x<+\infty\right\},
$$

and is endowed with the so-called Luxemburg norm

$$
|\mathbf{u}|_{p(x)}=\inf \left\{\lambda>0: \int_{\Omega}\left|\frac{\mathbf{u}}{\lambda}\right|^{p(x)} d x \leqslant 1\right\}
$$

If $p(x)=p \equiv$ constant for every $x \in \Omega$, then the $L^{p(x)}(\Omega)$ space is reduced to the classical Lebesgue space $L^{p}(\Omega)$ and the Luxemburg norm becomes the standard norm on $L^{p}(\Omega)$,

$$
\|\mathbf{u}\|_{L^{p}(\Omega)}=\left(\int_{\Omega}|\mathbf{u}(x)|^{p} d x\right)^{1 / p}
$$

If $p(x) \not \equiv$ constant in $\Omega$, then an important role in manipulating the generalized Lebesgue-Sobolev spaces is played by the modular $\rho_{p(\cdot)}$ of the space $L^{p(\cdot)}(\Omega)$, which is the mapping $\rho_{p(x)}: L^{p(x)}(\Omega) \rightarrow \mathbb{R}$ defined by

$$
\rho_{p(x)}(u):=\int_{\Omega}|u|^{p(x)} d x
$$


and the following properties hold:

$$
\begin{gathered}
|\mathbf{u}|_{p(x)}<1 \Rightarrow|\mathbf{u}|_{p(x)}^{p^{+}} \leqslant \rho_{p(x)}(\mathbf{u}) \leqslant|\mathbf{u}|_{p(x)}^{p^{-}}, \\
|\mathbf{u}|_{p(x)}>1 \Rightarrow|\mathbf{u}|_{p(x)}^{p^{-}} \leqslant \rho_{p(x)}(\mathbf{u}) \leqslant|\mathbf{u}|_{p(x)}^{p^{+}}, \\
|\mathbf{u}|_{p(x)}=1 \Rightarrow \rho_{p(x)}(\mathbf{u})=1, \\
\left|\mathbf{u}_{n}-\mathbf{u}\right|_{p(x)} \rightarrow 0 \Leftrightarrow \rho_{p(x)}\left(\mathbf{u}_{n}-\mathbf{u}\right) \rightarrow 0 .
\end{gathered}
$$

For more details about these variable exponent Lebesgue spaces see [17, 23, 37.

Remark 2.1. Variable exponent Lebesgue spaces resemble the classical Lebesgue spaces in many respects, they are separable Banach spaces and the Hölder inequality holds. The inclusions between Lebesgue spaces also naturally generalize, that is, if $0<\operatorname{meas}(\Omega)<\infty$ and $p(x), q(x)$ are variable exponents such that $p(x)<q(x)$ a.e. in $\Omega$, then there exists a continuous embedding $L^{q(x)}(\Omega) \hookrightarrow$ $L^{p(x)}(\Omega)$.

Definition 2.2. The variable exponent Sobolev space $W^{1, p(x)}$ is defined by

$$
W^{1, p(x)}(\Omega)=\left\{\mathbf{u} \in L^{p(x)}(\Omega):|\nabla \mathbf{u}| \in L^{p(x)}(\Omega)\right\},
$$

with the norm

$$
\begin{gathered}
\|\mathbf{u}\|_{1, p(x)}=\inf \left\{\lambda>0: \int_{\Omega}\left(\left|\frac{\nabla \mathbf{u}}{\lambda}\right|^{p(x)}+\left|\frac{\mathbf{u}}{\lambda}\right|^{p(x)}\right) d x \leqslant 1\right\}, \\
\|\mathbf{u}\|_{1, p(x)}=\|\nabla \mathbf{u}\|_{p(x)}+|\mathbf{u}|_{p(x)},
\end{gathered}
$$

where

$$
|\nabla \mathbf{u}|=\sqrt{\sum_{i=1}^{N}\left(\frac{\partial \mathbf{u}}{\partial x_{i}}\right)^{2}} .
$$

Definition 2.3. For $p(x) \in C_{+}(\bar{\Omega})$, let us define the so-called critical Sobolev exponent $p^{*}(x)$ of $p(x)$ by

$$
p^{*}(x)= \begin{cases}\frac{3 p(x)}{3-p(x)} & \text { if } p(x)<3, \\ +\infty & \text { if } p(x) \geqslant 3\end{cases}
$$

for every $x \in \bar{\Omega}$.

We define $W_{0}^{1, p(x)}(\Omega)$ as the closure of $C_{0}^{\infty}(\Omega)$ with respect to the norm $\|\cdot\|_{p(x)}$,

$$
W_{0}^{1, p(x)}(\Omega)=\left\{\mathbf{u}:\left.\mathbf{u}\right|_{\partial \Omega}=0, \mathbf{u} \in L^{p(x)}(\Omega),|\nabla \mathbf{u}| \in L^{p(x)}(\Omega)\right\} .
$$

The dual space of $W_{0}^{1, p(x)}(\Omega)$ is denoted by $W^{-1, p^{\prime}(x)}(\Omega)$, where

$$
\frac{1}{p(x)}+\frac{1}{p^{\prime}(x)}=1, \text { for every } x \in \bar{\Omega} .
$$

Next, we recall some embedding results regarding variable exponent Lebesgue and Sobolev spaces.

Theorem 2.2. (see [18, Theorem 1.3]) The following statements hold:

(i) $\left(W_{0}^{1, p(x)}(\Omega),\|\cdot\|\right)$ is a separable and reflexive Banach space.

(ii) If $p, q \in C_{+}(\bar{\Omega})$ and $q(x)<p^{*}(x)$ for every $x \in \bar{\Omega}$, then there is a compact and continuous embedding

$$
W^{1, p(x)}(\Omega) \hookrightarrow L^{q(x)}(\Omega) .
$$

(iii) There is a constant $C>0$ such that

$$
|\mathbf{u}|_{p(x)} \leqslant C\|\nabla \mathbf{u}\|_{p(x)}, \text { for all } \mathbf{u} \in W_{0}^{1, p(x)}(\Omega) .
$$


Let

and define

$$
\mathbf{L}^{p(x)}(\Omega)=L^{p(x)}(\Omega) \times L^{p(x)}(\Omega) \times L^{p(x)}(\Omega)
$$

$$
\mathbf{W}^{p(x)}(\Omega)=\left\{\mathbf{v} \in \mathbf{L}^{p(x)}(\Omega): \nabla \times \mathbf{v} \in \mathbf{L}^{p(x)}(\Omega), \nabla \cdot \mathbf{v}=0,\left.\mathbf{v} \cdot \mathbf{n}\right|_{\partial \Omega}=0\right\},
$$

where $\mathbf{n}$ denotes the outward unit normal vector to $\partial \Omega$. Equip $\mathbf{W}^{p(x)}(\Omega)$ with the norm

$$
\|\mathbf{v}\|_{\mathbf{W}^{p(x)}(\Omega)}=\|\mathbf{v}\|_{L^{p(x)}(\Omega)}+\|\nabla \times \mathbf{v}\|_{L^{p(x)}(\Omega)} .
$$

If $p^{-}>1$, then by Theorem 2.1 of $[6], \mathbf{W}^{p(x)}(\Omega)$ is a closed subspace of $\mathbf{W}_{\mathbf{n}}^{1, p(x)}(\Omega)$, where

$$
\mathbf{W}_{\mathbf{n}}^{1, p(x)}(\Omega)=\left\{\mathbf{v} \in W^{1, p(x)}(\Omega):\left.\mathbf{v} \cdot \mathbf{n}\right|_{\partial \Omega}=0\right\}
$$

and

$$
\mathbf{W}^{1, p(x)}(\Omega)=W^{1, p(x)}(\Omega) \times W^{1, p(x)}(\Omega) \times W^{1, p(x)}(\Omega) .
$$

Thus, we have the following theorem.

Theorem 2.3. (see [39, Theorem 2.1]) Assume that $1<p^{-} \leqslant p^{+}<\infty$ and $p$ satisfies condition 1.1). Then $\mathbf{W}^{p(x)}(\Omega)$ is a closed subspace of $\mathbf{W}_{n}^{1, p(x)}(\Omega)$. Moreover, if $p^{-}>\frac{6}{5}$, then $\|\nabla \times \cdot\|_{p(x)(\Omega)}$ is a norm on $\mathbf{W}^{p(x)}(\Omega)$ and there exists $C=C\left(N, p^{-}, p^{+}\right)>0$ such that

$$
\|\mathbf{v}\|_{W^{1, p(x)}(\Omega)} \leqslant C\|\nabla \times \mathbf{v}\|_{L^{p(x)}(\Omega)} .
$$

Remark 2.4. By Theorems 2.2 and 2.3, the embedding $\mathbf{W}^{p(x)}(\Omega) \hookrightarrow \mathbf{L}^{q(x)}(\Omega)$ is compact, with $1<p^{-} \leqslant p^{+}<3, q \in C(\bar{\Omega})$, and $1 \leqslant q(x)<\frac{3 p(x)}{3-p(x)}$ in $\bar{\Omega}$. Moreover, $\left(\mathbf{W}^{p(x)}(\Omega),\|\cdot\|\right)$ is a uniformly convex, reflexive and separable Banach space.

Let

$$
\|\mathbf{u}\|_{a}=\inf \left\{\eta>0: \int_{\Omega}\left(\left|\frac{\nabla \times \mathbf{u}(x)}{\eta}\right|^{p(x)}+a(x)\left|\frac{\mathbf{u}(x)}{\eta}\right|^{p(x)}\right) d x \leqslant 1\right\}
$$

for all $\mathbf{u} \in \mathbf{W}^{p(x)}$. In view of $a^{-}>0$ (see condition $\left.(\mathcal{A})_{1}\right)$, it is easy to see that $\|\cdot\|_{a}$ is equivalent to the norms $\|\cdot\|_{\mathbf{W}^{p(x)}(\Omega)}$ and $\|\cdot\|_{L^{p(x)}(\Omega)}$ on $\mathbf{W}^{p(x)}(\Omega)$. In this paper, we shall use for convenience the norm $\|\cdot\|_{a}$ on the space $\mathbf{W}^{p(x)}(\Omega)$.

Proposition 2.5. (see [39]) Let

$$
\Lambda_{p(x), a}(\mathbf{u})=\int_{\Omega}\left(|\nabla \times \mathbf{u}(x)|^{p(x)}+a(x)|\mathbf{u}(x)|^{p(x)}\right) d x \text { for all } \mathbf{u} \in \mathbf{W}^{p(x)}(\Omega) .
$$

Then

(1) $|\mathbf{u}|_{a}<1 \Rightarrow|\mathbf{u}|_{a}^{p^{+}} \leqslant \Lambda_{p(x), a}(\mathbf{u}) \leqslant|\mathbf{u}|_{a}^{p^{-}}$

(2) $|\mathbf{u}|_{a}>1 \Rightarrow|\mathbf{u}|_{a}^{p^{-}} \leqslant \Lambda_{p(x), a}(\mathbf{u}) \leqslant|\mathbf{u}|_{a}^{p^{+}}$.

Proposition 2.6. The following functional

$$
\Phi(\mathbf{u})=\int_{\Omega} \frac{1}{p(x)}\left(|\nabla \times \mathbf{u}(x)|^{p(x)}+a(x)|\mathbf{u}(x)|^{p(x)}\right) d x,
$$

is well defined, even, convex, and sequentially weakly lower semi-continuous. Also, the functional $\Phi$ is of class $C^{1}$ and

$$
\left(\Phi^{\prime}(\mathbf{u}), \mathbf{v}\right)=\int_{\Omega}\left(|\nabla \times \mathbf{u}|^{p(x)-2} \nabla \times \mathbf{u} \cdot \nabla \times \mathbf{v}+a(x)|\mathbf{u}|^{p(x)-2} \mathbf{u} \cdot \mathbf{v}\right) d x, \text { for all } \mathbf{u}, \mathbf{v} \in \mathbf{W}^{p(x)}(\Omega)
$$

where $\langle\cdot, \cdot\rangle$ is the dual pairing between $\mathbf{W}^{p(x)}(\Omega)$ and its dual $\left(\mathbf{W}^{p(x)}(\Omega)\right)^{*}$. Similar to [39], we can deduce that

(i) $\Phi^{\prime}: \mathbf{W}^{p(x)}(\Omega) \rightarrow\left(\mathbf{W}^{p(x)}(\Omega)\right)^{*}$ is a continuous, bounded and strictly monotone operator;

(ii) $\Phi^{\prime}$ is a mapping of type $\left(S_{+}\right)$, namely: $\mathbf{u}_{n} \rightarrow \mathbf{u}$ and $\limsup _{n \rightarrow \infty}\left\langle A^{\prime}\left(\mathbf{u}_{n}\right), \mathbf{u}_{n}-\mathbf{u}\right\rangle \leqslant 0$, hence $\mathbf{u}_{n} \rightarrow \mathbf{u}$ in $\mathbf{W}^{p(x)}(\Omega)$. 
(iii) $\Phi^{\prime}: \mathbf{W}^{p(x)}(\Omega) \rightarrow\left(\mathbf{W}^{p(x)}(\Omega)\right)^{*}$ is a homeomorphism.

Remark 2.7. (see [20, Remark 2.1]) We note that the sum of a mapping of type $\left(S_{+}\right)$and a weaklystrongly continuous mapping is still a mapping of type $\left(S_{+}\right)$. Therefore $I_{\lambda, \mu}^{\prime}=\Phi^{\prime}-\lambda J^{\prime}-\mu \Psi^{\prime}$ is a mapping of type $\left(S_{+}\right)$. Hence any bounded $(P . S)$ sequence of $I_{\lambda, \mu}$ has a convergent subsequence.

2.2. Preliminary lemmas. From the statement above we know that $\mathbf{W}^{p(x)}(\Omega)$ is a reflexive and separable Banach space (see [6]). Therefore there exist $\left\{e_{j}\right\} \subset \mathbf{W}^{p(x)}(\Omega)$ and $\left\{e_{j}^{*}\right\} \subset\left(\mathbf{W}^{p(x)}(\Omega)\right)^{*}$ such that

$$
\mathbf{W}^{p(x)}(\Omega)=\overline{\operatorname{span}}\left\{e_{j}: j=1,2, \ldots\right\},\left(\mathbf{W}^{p(x)}(\Omega)\right)^{*}=\overline{\operatorname{span}}\left\{e_{j}^{*}: j=1,2, \ldots\right\},
$$

with

$$
\left\langle e_{j}, e_{j}^{*}\right\rangle= \begin{cases}1, & \text { if } i=j \\ 0, & \text { if } i \neq j\end{cases}
$$

Define

$$
X_{j}=\operatorname{span}\left\{e_{j}\right\}, Y_{k}=\bigoplus_{j=1}^{k} X_{j}, Z_{k}=\bigoplus_{j=k}^{\infty} X_{j} .
$$

We need the following lemmas which will be used in the proof of our main results.

Lemma 2.8. If $q(x), \gamma(x) \in \mathcal{C}_{+}(\bar{\Omega}), q(x), \gamma(x)<p_{2}^{*}(x)$ for $x \in \bar{\Omega}$, let

$$
\begin{aligned}
& \beta_{k}=\sup \left\{|\mathbf{u}|_{q(x)}:\|\mathbf{u}\|_{a}=1, \mathbf{u} \in Z_{k}\right\}, \\
& \theta_{k}=\sup \left\{|\mathbf{u}|_{\gamma(x)}:\|\mathbf{u}\|_{a}=1, u \in Z_{k}\right\} .
\end{aligned}
$$

Then $\lim _{k \rightarrow \infty} \beta_{k}=0$ and $\lim _{k \rightarrow \infty} \theta_{k}=0$.

Proof. Obviously, $0<\beta_{k+1} \leqslant \beta_{k}$, so $\beta_{k} \rightarrow \beta \geqslant 0$. Let $\mathbf{u}_{k} \in Z_{k}$ satisfy

$$
\left\|\mathbf{u}_{k}\right\|_{a}=1, \quad 0 \leqslant \beta_{k}-\left|\mathbf{u}_{k}\right|_{q(x)}<\frac{1}{k} .
$$

Then there exists a subsequence of $\left\{\mathbf{u}_{k}\right\}$ (which we still denote by $\mathbf{u}_{k}$ ) such that $\mathbf{u}_{k} \rightarrow \mathbf{u}$, and

$$
\left\langle e_{j}^{*}, u\right\rangle=\lim _{k \rightarrow \infty}\left\langle e_{j}^{*}, \mathbf{u}_{k}\right\rangle=0 \text {, for all } e_{j}^{*},
$$

which implies that $\mathbf{u}=0$, and so $\mathbf{u}_{k} \rightarrow 0$. Since the embedding from $\mathbf{W}^{p(x)}(\Omega)$ to $\mathbf{L}^{q(x)}(\Omega)$ is compact, it follows that $\mathbf{u}_{k} \rightarrow 0$ in $\mathbf{L}^{q(x)}(\Omega)$. Hence, we get $\beta_{k} \rightarrow 0$ as $k \rightarrow \infty$. The proof for $\theta_{k}$ can be obtained by the same procedure.

Lemma 2.9. $I_{\lambda, \mu}$ is weakly lower semi-continuous on $\mathbf{W}^{p(x)}(\Omega)$.

Proof By Proposition 2.6 we know that $\Phi$ is weakly lower semi-continuous. Assuming $\mathbf{u}_{n} \rightarrow \mathbf{u}$ in $\mathbf{W}^{p(x)}(\Omega)$, the compact embedding by Remark 2.4 gives us

$$
\mathbf{u}_{n} \rightarrow \mathbf{u} \text { in } \mathbf{L}^{p(x)}(\Omega) \text { and } \mathbf{u}_{n} \rightarrow \mathbf{u} \text { in } \mathbf{L}^{1}(\Omega) .
$$

By the mean value theorem, there exists $\mathbf{z}$ which takes on values strictly between $\mathbf{u}$ and $\mathbf{u}_{n}$ such that

$$
\int_{\Omega}\left|F\left(x, \mathbf{u}_{n}\right)-F(x, \mathbf{u})\right| d x \leqslant \int_{\Omega}\left|\mathbf{u}_{n}-\mathbf{u}\right| \sup _{x \in \Omega}|f(x, \mathbf{z})| d x,
$$

hence by assumptions $\left.\left(f_{2}\right), g_{2}\right)$ and $(2.2)$, the functional $J(\mathbf{u})=\int_{\Omega} F(x, \mathbf{u}) d x$ is weakly continuous, and so is $\Psi(\mathbf{u})=\int_{\Omega} G(x, \mathbf{u}) d x$. Consequently, the functional $I_{\lambda, \mu}$ is weakly lower semi-continuous. 


\section{Proofs of the main Results}

Recall the definitions of $(P S)_{c}$ and $(P S)_{c}^{*}$ conditions ${ }^{1}$

\subsection{The Palais-Smale Compactness Condition.}

Definition 3.1. The $C^{1}$-functional $I_{\lambda, \mu}$ satisfies the Palais-Smale condition at the level $c$ (in short $(P S)_{c}$ condition) for $c \in \mathbb{R}$ if any sequence $\left(\mathbf{u}_{n}\right)_{n \in \mathbb{N}} \subseteq \mathbf{W}^{p(x)}(\Omega)$ for which $I_{\lambda, \mu}\left(\mathbf{u}_{n}\right) \rightarrow c$ and $I_{\lambda, \mu}^{\prime}\left(\mathbf{u}_{n}\right) \rightarrow 0$ as $n \rightarrow \infty$, has a convergent subsequence.

Definition 3.2. The $C^{1}$-functional $I_{\lambda, \mu}$ satisfies the $(P S)_{c}^{*}$ condition for $c \in \mathbb{R}$ if any sequence $\left(\mathbf{u}_{n}\right)_{n \in \mathbb{N}} \subseteq \mathbf{W}^{p(x)}(\Omega)$ such that $n_{j} \rightarrow+\infty, \mathbf{u}_{n_{j}} \in Y_{n_{j}}, I_{\lambda, \mu}\left(\mathbf{u}_{n_{j}}\right) \rightarrow c$, and $\left(I_{\lambda, \mu} \mid Y_{n_{j}}\right)^{\prime}\left(\mathbf{u}_{n_{j}}\right) \rightarrow 0$, contains a subsequence converging to a critical point of $I_{\lambda, \mu}$.

Theorem 3.1. Under the hypotheses of Theorem 1.4, the functional $I_{\lambda, \mu}$ satisfies $(P S)_{c}$ condition.

Proof. By Lemma 2.9 and Remark 2.7, it suffices to verify the boundedness of $(P S)_{c}$ sequences. Suppose that $\left(\mathbf{u}_{n}\right)_{n} \subset \mathbf{W}^{p(x)}(\Omega)$ is a $(P S)$ sequence at the level $c \in \mathbb{R}$, i.e., $I_{\lambda, \mu}\left(\mathbf{u}_{n}\right) \leqslant c$ and $I_{\lambda, \mu}^{\prime}\left(\mathbf{u}_{n}\right) \rightarrow 0$ as $n \rightarrow \infty$. Arguing by contradiction, we assume that $\left\|\mathbf{u}_{n}\right\|_{a} \rightarrow+\infty$. For $n$ large enough, by the conditions $\left(f_{1}, f_{3}, g_{1}, g_{2}\right.$ and Proposition 2.5 we have

$$
\begin{aligned}
c+\left\|\mathbf{u}_{n}\right\|_{a} \geqslant & I_{\lambda, \mu}\left(\mathbf{u}_{n}\right)-\frac{1}{\mu}\left\langle I_{\lambda, \mu}^{\prime}\left(\mathbf{u}_{n}\right), \mathbf{u}_{n}\right\rangle \\
\geqslant & \left(\frac{1}{p^{+}}-\frac{1}{\mu}\right) \Lambda_{p(x), a}(\mathbf{u})-\mu \int_{\Omega}\left(\frac{1}{\mu} G\left(x, \mathbf{u}_{n}\right)-g\left(x, \mathbf{u}_{n}\right) \cdot \mathbf{u}_{n}\right) d x \\
& +\lambda \int_{\Omega}\left(\frac{1}{\mu} f\left(x, \mathbf{u}_{n}\right) \cdot \mathbf{u}_{n}-F\left(x, \mathbf{u}_{n}\right)\right) d x \\
\geqslant & \left(\frac{1}{p^{+}}-\frac{1}{\mu}\right)\left\|\mathbf{u}_{n}\right\|_{a}^{p^{-}}-\mu \int_{\Omega}\left(\frac{1}{\mu} G\left(x, \mathbf{u}_{n}\right)-g\left(x, \mathbf{u}_{n}\right) \cdot \mathbf{u}_{n}\right) d x \\
& +\lambda \int_{\Omega \cap\left\{\left|\mathbf{u}_{n}\right|>l\right\}}\left(\frac{1}{\mu} f\left(x, \mathbf{u}_{n}\right) \cdot \mathbf{u}_{n}-F\left(x, \mathbf{u}_{n}\right)\right) d x-C|\Omega| \\
\geqslant & \left(\frac{1}{p^{+}}-\frac{1}{\mu}\right)\left\|\mathbf{u}_{n}\right\|_{a}^{p^{-}}-C \mu\left\|\mathbf{u}_{n}\right\|_{a}^{\gamma^{+}}-C|\Omega| .
\end{aligned}
$$

Dividing the above inequality by $\left\|\mathbf{u}_{n}\right\|_{a}^{p^{-}}$, taking into account that $p^{-}>\gamma^{+}$and passing to the limit as $n \rightarrow \infty$, we obtain a contradiction. It follows that $\left(\mathbf{u}_{n}\right)_{n}$ is bounded in $\mathbf{W}^{p(x)}(\Omega)$.

Theorem 3.2. Under the hypotheses of Theorem 1.5, functional $I_{\lambda, \mu}$ satisfies $(P S)_{c}^{*}$ condition.

Proof. Suppose that $\left(\mathbf{u}_{n_{j}}\right)_{j} \subset \mathbf{W}^{p(x)}(\Omega)$ is such that

$$
\mathbf{u}_{n_{j}} \in Y_{n_{j}}, I_{\lambda, \mu}\left(\mathbf{u}_{n_{j}}\right) \rightarrow c,\left(I_{\lambda, \mu} \mid Y_{n_{j}}\right)^{\prime}\left(\mathbf{u}_{n_{j}}\right) \rightarrow 0 \text { as } n_{j} \rightarrow+\infty
$$

In a similar way as in the proof of Theorem 3.1 we obtain the boundedness of the sequence $\left(\mathbf{u}_{n_{j}}\right)_{j \in \mathbb{N}^{*}} \subseteq \mathbf{W}^{p(x)}(\Omega)$. Hence, there exists $\mathbf{u} \in \mathbf{W}^{p(x)}(\Omega)$ such that $\mathbf{u}_{n_{j}} \rightarrow \mathbf{u}$ weakly in $\mathbf{W}^{p(x)}(\Omega)=$ $\overline{\cup_{n_{j}} Y_{n_{j}}}$. Then we can obtain $\mathbf{v}_{n_{j}} \in Y_{n_{j}}$ such that $\mathbf{v}_{n_{j}} \rightarrow \mathbf{u}$. We have

$$
\left\langle I_{\lambda, \mu}^{\prime}\left(\mathbf{u}_{n_{j}}\right), \mathbf{u}_{n_{j}}-\mathbf{u}\right\rangle=\left\langle I_{\lambda, \mu}^{\prime}\left(\mathbf{u}_{n_{j}}\right), \mathbf{u}_{n_{j}}-\mathbf{v}_{n_{j}}\right\rangle+\left\langle I_{\lambda, \mu}^{\prime}\left(\mathbf{u}_{n_{j}}\right), \mathbf{v}_{n_{j}}-\mathbf{u}\right\rangle .
$$

Since $\mathbf{u}_{n_{j}}-\mathbf{v}_{n_{j}} \in Y_{n_{j}}$, then

$$
\left\langle I_{\lambda, \mu}^{\prime}\left(\mathbf{u}_{n_{j}}\right), \mathbf{u}_{n_{j}}-\mathbf{u}\right\rangle=\left\langle\left(I_{\lambda, \mu} \mid Y_{n_{j}}\right)^{\prime}\left(\mathbf{u}_{n_{j}}\right), \mathbf{u}_{n_{j}}-\mathbf{v}_{n_{j}}\right\rangle+\left\langle I_{\lambda, \mu}^{\prime}\left(\mathbf{u}_{n_{j}}\right), \mathbf{v}_{n_{j}}-\mathbf{u}\right\rangle \rightarrow 0 \text { as } n \rightarrow \infty .
$$

\footnotetext{
${ }^{1}$ We refer the readers to [24, 25, 26, 28, for further information on the Palais-Smale condition with assumption weaker than $f_{3}$.
} 
Since $I_{\lambda, \mu}^{\prime}$ is of $S_{+}$type, we can deduce that $\mathbf{u}_{n_{j}} \rightarrow \mathbf{u}$ in $\mathbf{W}^{p(x)}(\Omega)$. Furthermore, $I_{\lambda, \mu}^{\prime}\left(\mathbf{u}_{n_{j}}\right) \rightarrow$ $I_{\lambda, \mu}^{\prime}(\mathbf{u})$. Now we claim that $\mathbf{u}$ is a critical point of $I_{\lambda, \mu}$. Taking $\mathbf{w}_{k} \in Y_{k}$, when $n_{j} \geqslant k$, we have

$$
\begin{aligned}
& \left\langle I_{\lambda, \mu}^{\prime}(\mathbf{u}), \mathbf{w}_{k}\right\rangle=\left\langle I_{\lambda, \mu}^{\prime}(\mathbf{u})-I_{\lambda, \mu}^{\prime}\left(\mathbf{u}_{n_{j}}\right), \mathbf{w}_{k}\right\rangle+\left\langle I_{\lambda, \mu}^{\prime}\left(\mathbf{u}_{n_{j}}\right), \mathbf{w}_{k}\right\rangle \\
& =\left\langle I_{\lambda, \mu}^{\prime}(\mathbf{u})-I_{\lambda, \mu}^{\prime}\left(\mathbf{u}_{n_{j}}\right), \mathbf{w}_{k}\right\rangle+\left\langle\left(I_{\lambda, \mu} \mid Y_{n_{j}}\right)^{\prime}\left(\mathbf{u}_{n_{j}}\right), \mathbf{w}_{k}\right\rangle .
\end{aligned}
$$

Taking $n_{j} \rightarrow \infty$, we obtain $\left\langle I_{\lambda, \mu}^{\prime}(\mathbf{u}), \mathbf{w}_{k}\right\rangle=0$, for all $\mathbf{w}_{k} \in Y_{k}$. So $I_{\lambda, \mu}^{\prime}(\mathbf{u})=0$, which verifies that $I_{\lambda, \mu}$ satisfies $(P S)_{c}^{*}$ condition.

3.2. Proof of Theorem 1.4. The following Fountain theorem will be used to get our first result. For the reader convenience, we state it as follows.

Theorem 3.3. (Fountain theorem [40]) Let $X$ be a reflexive and separable Banach space, $I \in$ $C^{1}(X, \mathbb{R})$ an even functional and let the subspaces $X_{k}, Y_{k}, Z_{k}$ be as defined in (2.1). Suppose that for each $k \in \mathbb{R}$, there exist $\rho_{k}>r_{k}>0$ such that

$\left(A_{1}\right) \inf \left\{I(\mathbf{u}): \mathbf{u} \in Z_{k},\|\mathbf{u}\|=r\right\} \rightarrow+\infty$ as $k \rightarrow+\infty$;

$\left(A_{2}\right) \max \left\{I(\mathbf{u}): \mathbf{u} \in Y_{k},\|\mathbf{u}\|=\rho_{k}\right\} \leqslant 0$

$\left(A_{3}\right) I$ satisfies $(P S)$ condition for every $c>0$,

Then I has an unbounded sequence of critical points.

Proof of Theorem 1.4. By $\left(g_{3}, f_{4}\right)$ and Theorem 3.1, $I_{\lambda, \mu}$ is an even functional and satisfies $(P S)_{c}$ condition. Therefore, by Theorem 3.3 it suffices to show that if $k$ is large enough, then there exist $\rho_{k}>r_{k}>0$ such that $\left(A_{1}\right)$ and $\left(A_{2}\right)$ hold.

Verification of $\left(\mathbf{A}_{\mathbf{1}}\right)$ : Let $\mathbf{u} \in Z_{k}$ with $\|u\|_{a}>1$. Then it follows from $\left[f_{2}\right.$ and $g_{2}$ that

$$
\begin{aligned}
I_{\lambda, \mu}(\mathbf{u}) & =\int_{\Omega} \frac{1}{p(x)}\left(|\nabla \times \mathbf{u}(x)|^{p(x)}+a(x)|\mathbf{u}(x)|^{p(x)}\right) d x-\lambda \int_{\Omega} F(x, \mathbf{u}) d x-\mu \int_{\Omega} G(x, \mathbf{u}) d x \\
& \geqslant \frac{1}{p^{+}}\|\mathbf{u}\|_{a}^{p^{-}}-\lambda \int_{\Omega} F(x, \mathbf{u}) d x-\mu \int_{\Omega} G(x, \mathbf{u}) d x \\
& \geqslant \frac{1}{p^{+}}\|\mathbf{u}\|_{a}^{p^{-}}-\lambda C \int_{\Omega}|\mathbf{u}|^{q(x)} d x-\mu C \int_{\Omega}|\mathbf{u}|^{\gamma(x)} d x \\
& \geqslant \frac{1}{p^{+}}\|\mathbf{u}\|_{a}^{p^{-}}-\lambda C \rho_{q(x)}(\mathbf{u})-\mu C\|\mathbf{u}\|_{a}^{\gamma^{+}} \\
& \geqslant\left\{\begin{array}{l}
\frac{1}{p^{+}}\|\mathbf{u}\|_{a}^{p^{-}}-C-\mu C\|\mathbf{u}\|_{a}^{\gamma^{+}}, \quad \text { if }|\mathbf{u}|_{\gamma(x)} \leqslant 1 \\
\frac{1}{p^{+}}\|\mathbf{u}\|_{a}^{p^{-}}-\frac{\lambda}{q^{-}} C \beta_{k}^{q^{+}}\|\mathbf{u}\|_{a}^{q^{+}}-\mu C\|\mathbf{u}\|_{a}^{\gamma^{+}}, \quad \text { if }|\mathbf{u}|_{\gamma(x)}>1 . \\
\end{array} \frac{1}{p^{+}}\|\mathbf{u}\|_{a}^{p^{-}}-\frac{\lambda}{q^{-}} C \beta_{k}^{q^{+}}\|\mathbf{u}\|_{a}^{q^{+}}-\mu C\|\mathbf{u}\|_{a}^{\gamma^{+}}-C,\right.
\end{aligned}
$$

where

$$
\beta_{k}=\sup \left\{|\mathbf{u}|_{q(x)}:\|\mathbf{u}\|_{a}=1, \mathbf{u} \in Z_{k}\right\} .
$$

Choose $\|\mathbf{u}\|_{a}=r_{k}=\left(\frac{\lambda}{q^{-}} q^{+} C \beta_{k}^{q^{+}}\right)^{\frac{1}{p^{--q^{+}}}}$and notice that $p^{-}<p^{+}<q^{+}$. By Lemma 2.8 we can deduce that $r_{k} \rightarrow+\infty$ as $k \rightarrow \infty$, hence

$$
\begin{aligned}
I_{\lambda, \mu}(\mathbf{u}) & \geqslant \frac{1}{p^{+}}\left(\frac{\lambda}{q^{-}} q^{+} C \beta_{k}^{q^{+}}\right)^{\frac{p^{-}}{p^{-}-q^{+}}}-\frac{1}{q^{+}}\left(\frac{\lambda}{q^{-}} q^{+} C \beta_{k}^{q^{+}}\right)\left(\frac{\lambda}{q^{-}} q^{+} C \beta_{k}^{q^{+}}\right)^{\frac{q^{+}}{p^{-}-q^{+}}}-\mu C\left(\frac{\lambda}{q^{-}} q^{+} C \beta_{k}^{q^{+}}\right)^{\frac{\gamma^{+}}{p^{-}-q^{+}}}-C . \\
& =\left(\frac{1}{p^{+}}-\frac{1}{q^{+}}\right)\left(\frac{\lambda}{q^{-}} q^{+} C \beta_{k}^{q^{+}}\right)^{\frac{p^{-}}{p-q^{+}}}-\mu C\left(\frac{\lambda}{q^{-}} q^{+} C \beta_{k}^{q^{+}}\right)^{\frac{\gamma^{+}}{p^{-}-q^{+}}}-C \rightarrow \infty \text { as } k \rightarrow \infty .
\end{aligned}
$$

Verification of $\left(\mathbf{A}_{\mathbf{2}}\right)$ : Clearly, condition $\left(f_{3}\right)$ implies the existence of two positive constants $c_{1}$ and $c_{2}$ such that

$$
F(x, u) \geqslant c_{1}|\mathbf{u}|^{\theta}-c_{2}, \text { for all }(x, \mathbf{u}) \in \Omega \times \mathbb{R}^{3} .
$$


Assume now that (3.1) and $\left(g_{2}\right)$ hold. Let $\mathbf{u} \in Y_{k}$ be such that $\|\mathbf{u}\|_{a}=\rho_{k}>r_{k}>1$. Then

$$
\begin{aligned}
I_{\lambda, \mu}(\mathbf{u}) & =\int_{\Omega} \frac{1}{p(x)}\left(|\nabla \times \mathbf{u}(x)|^{p(x)}+a(x)|\mathbf{u}(x)|^{p(x)}\right) d x-\lambda \int_{\Omega} F(x, \mathbf{u}) d x-\mu \int_{\Omega} G(x, \mathbf{u}) d x \\
& \leqslant \frac{1}{p^{-}}\|\mathbf{u}\|_{a}^{p^{+}}-\lambda \int_{\Omega} F(x, \mathbf{u}) d x-\mu \int_{\Omega} G(x, \mathbf{u}) d x \\
& \leqslant \frac{1}{p^{-}}\|\mathbf{u}\|_{a}^{p^{+}}-\lambda c_{1} \int_{\Omega}|u|^{\theta} d x+\mu C \int_{\Omega}|\mathbf{u}|^{\gamma(x)} d x+c_{2}|\Omega| .
\end{aligned}
$$

Since $\operatorname{dim} Y_{k}<\infty$, all norms are equivalent in $Y_{k}$, there are $C_{W}^{1}, C_{W}^{2}>0$ such that

$$
\int_{\Omega}|u|^{\theta} d x \geqslant C_{W}^{1}\|u\|_{a}^{\theta} \text { and } \int_{\Omega}|u|^{\gamma(x)} d x \leqslant C_{W}^{2}\|u\|_{a}^{\gamma^{+}} .
$$

Hence, we get

$$
I_{\lambda, \mu}(\mathbf{u}) \leqslant \frac{1}{p^{-}}\|\mathbf{u}\|_{a}^{p^{+}}-\lambda c_{1} C_{W}^{1} \lambda\|\mathbf{u}\|_{a}^{\theta}+\mu C C_{W}^{2}\|\mathbf{u}\|_{a}^{\gamma^{+}}+c_{2}|\Omega|,
$$

so we see that $I_{\lambda, \mu}(\mathbf{u}) \rightarrow-\infty$ as $\|\mathbf{u}\|_{a} \rightarrow+\infty$ because $\gamma^{+}<p^{+}<\theta$. Conclusion of Theorem 1.4 is now reached by invoking Theorem 3.3 .

3.3. Proof of Theorem 1.5. We shall apply the following Dual Fountain theorem to prove our second main result.

Theorem 3.4. (Dual Fountain theorem [40) Let $X$ be a reflexive and separable Banach space, $I \in C^{1}(X, \mathbb{R})$ an even functional, and $X_{k}, Y_{k}, Z_{k}$ the subspaces defined in 2.1). Assume that there is $k_{0}>0$ such that for each $k>k_{0}$, there exist $\rho_{k}>r_{k}>0$ such that

$\left(B_{1}\right) \inf \left\{I(\|\mathbf{u}\|): \mathbf{u} \in Z_{k},\|\mathbf{u}\|=r_{k}\right\}<0$

$\left(B_{2}\right) \max \left\{I(\mathbf{u}): \mathbf{u} \in Y_{k},\|\mathbf{u}\|=\rho_{k}\right\} \geqslant 0$

$\left(B_{3}\right) \inf \left\{I(\mathbf{u}): \mathbf{u} \in Z_{k},\|\mathbf{u}\|=\rho_{k}\right\} \rightarrow 0$ as $k \rightarrow+\infty$;

$\left(B_{4}\right)$ I satisfies $(P S)_{c}^{*}$ condition for every $c \in\left[d_{k_{0}}, 0\right)$.

Then $I$ has a sequence of negative critical values converging to 0 .

Proof of Theorem 1.5. According to $\left(g_{3}\right), \sqrt{\left.f_{4}\right]}$ and Theorem $3.2, I_{\lambda, \mu}$ is an even functional and satisfies $(P S)_{c}^{*}$ condition. Thus it suffices to verify $\left(\mathbf{B}_{\mathbf{1}}\right),\left(\mathbf{B}_{\mathbf{2}}\right)$ and $\left(\mathbf{B}_{\mathbf{3}}\right)$ of Theorem 3.4 .

Verification of $\left(\mathbf{B}_{\mathbf{1}}\right)$ : Assume that $\left(f_{2}\right)$ and $g_{2}$ hold. For any $\mathbf{u} \in Z_{k}$, we have

$$
\begin{aligned}
I_{\lambda, \mu}(\mathbf{u}) & =\int_{\Omega} \frac{1}{p(x)}\left(|\nabla \times \mathbf{u}(x)|^{p(x)}+a(x)|\mathbf{u}(x)|^{p(x)}\right) d x-\lambda \int_{\Omega} F(x, \mathbf{u}) d x-\mu \int_{\Omega} G(x, \mathbf{u}) d x \\
& \geqslant \frac{1}{p^{+}}\|\mathbf{u}\|_{a}^{p^{+}}-\lambda \int_{\Omega} F(x, \mathbf{u}) d x-\mu \int_{\Omega} G(x, \mathbf{u}) d x \\
& \geqslant \frac{1}{p^{+}}\|\mathbf{u}\|_{a}^{p^{+}}-\lambda C\|\mathbf{u}\|_{a}^{q^{-}}-C \mu \int_{\Omega}|\mathbf{u}|^{\gamma(x)} d x .
\end{aligned}
$$

Notice that $q^{-}>p^{+}$, so there exists small enough $\rho_{0}>0$ such that $\lambda C\|\mathbf{u}\|_{a}^{q^{-}} \leqslant \frac{1}{2 p^{+}}\|\mathbf{u}\|_{a}^{p^{+}}$as $0<\rho=\|\mathbf{u}\|_{a} \leqslant \rho_{0}$.

Then by the proof above, we have

$$
I_{\lambda, \mu}(\mathbf{u}) \geqslant \begin{cases}\frac{1}{2 p^{+}}\|\mathbf{u}\|_{a}^{p^{+}}-\mu C \theta_{k}^{\gamma^{-}}\|\mathbf{u}\|_{a}^{\gamma^{-}}, & \text {if }|\mathbf{u}|_{\gamma(x)} \leqslant 1 \\ \frac{1}{2 p^{+}}\|\mathbf{u}\|_{a}^{p^{+}}-\mu C \theta_{k}^{\gamma^{-}}\|\mathbf{u}\|_{a}^{\gamma^{+}}, & \text {if }|\mathbf{u}|_{\gamma(x)}>1 .\end{cases}
$$

Choose

$$
\rho_{k}=\max \left\{\left(2 p^{+} C \mu \theta_{k}^{\gamma^{-}}\right)^{\frac{1}{p^{+}-\gamma^{-}}},\left(2 p^{+} C \mu \theta_{k}^{\gamma^{+}}\right)^{\frac{1}{p^{+}-\gamma^{+}}}\right\},
$$

and notice that $p^{+}>\gamma^{+}$, so by Lemma2.8 we can deduce that $\rho_{k} \rightarrow 0$ as $k \rightarrow \infty$. Hence $I_{\lambda, \mu}(\mathbf{u}) \geqslant 0$, i.e., $\left(\mathbf{B}_{\mathbf{1}}\right)$ is satisfied. 
Verification of $\left(\mathbf{B}_{\mathbf{2}}\right)$ : Assume that $\mathbf{u} \in Y_{k}$ with $\|\mathbf{u}\|_{a} \leqslant 1$. Assumption $\left(g_{4}\right)$ is equivalent to the following

$$
\text { there exists } \delta>0, G(x, \mathbf{t}) \geqslant C|\mathbf{t}|^{\alpha}, \alpha<p^{-}, \text {for all }|\mathbf{t}| \in(0, \delta) .
$$

Then by (3.4) and $\left(f_{2}\right)$ we have

$$
\begin{aligned}
I_{\lambda, \mu}(\mathbf{u}) & =\int_{\Omega} \frac{1}{p(x)}\left(|\nabla \times \mathbf{u}(x)|^{p(x)}+a(x)|\mathbf{u}(x)|^{p(x)}\right) d x-\lambda \int_{\Omega} F(x, \mathbf{u}) d x-\mu \int_{\Omega} G(x, \mathbf{u}) d x \\
& \leqslant \frac{1}{p^{-}}\|\mathbf{u}\|_{a}^{p^{-}}+\lambda C \int_{\Omega}|\mathbf{u}|^{q(x)} d x-\mu C \int_{\Omega}|\mathbf{u}|^{\alpha} d x \\
& \leqslant \frac{1}{p^{-}}\|\mathbf{u}\|_{a}^{p^{-}}+C\|\mathbf{u}\|_{a}^{q^{-}}-\mu C\|\mathbf{u}\|_{a}^{\alpha} .
\end{aligned}
$$

Since $\alpha<p^{-}<q^{-}$, there exists $r_{k} \in\left(0, \rho_{k}\right)$ such that $I_{\lambda, \mu}(u)<0$ when $\|\mathbf{u}\|_{a}=r_{k}$.

Verification of $\left(\mathbf{B}_{3}\right)$ : Notice that $Y_{k} \cap Z_{k} \neq \emptyset$ and $r_{k}<\rho_{k}$, so we have

$$
d_{k}=\inf _{\mathbf{u} \in Z_{k},\|\mathbf{u}\|_{a} \leqslant \rho_{k}} I_{\lambda, \mu}(\mathbf{u}) \leqslant b_{k}=\max _{\mathbf{u} \in Y_{k},\|\mathbf{u}\|_{a}=r_{k}} I_{\lambda, \mu}(\mathbf{u})<0 .
$$

For $\mathbf{u} \in Z_{k},\|\mathbf{u}\|_{a} \leqslant \rho_{k}$ is small enough. From (3.3), we can now obtain

$$
I_{\lambda, \mu}(\mathbf{u}) \geqslant \frac{1}{2 p^{+}}\|\mathbf{u}\|_{a}^{p^{+}}-\mu C \theta_{k}^{\gamma^{+}}\|\mathbf{u}\|_{a}^{\gamma^{+}} .
$$

Since $\theta_{k} \rightarrow 0$ and $k \rightarrow \infty$, it now follows that $\left(\mathbf{B}_{\mathbf{3}}\right)$ is also satisfied. Invoking Theorem 3.4 . we thus complete the proof of Theorem 1.5 .

3.4. Proof of Theorem 1.6. In order to prove Theorem 1.6, we shall need the following two lemmas.

Lemma 3.5. For any $\lambda\langle 0, \mu\rangle 0$, the following holds:

(1) $I_{\lambda, \mu}$ is weakly lower semi-continuous on $\mathbf{W}^{p(x)}(\Omega)$.

(2) $I_{\lambda, \mu}$ is bounded from below and coercive on $\mathbf{W}^{p(x)}(\Omega)$.

\section{Proof.}

(1) The proof is similar to that of Lemma 2.9, so we shall omit it.

(2) From the hypothese $\left[f_{5}\right]$, for any small enough $\epsilon>0$, there exists $M>0$ such that

$$
|F(x, \mathbf{t})| \leqslant \epsilon|\mathbf{t}|^{p^{-}} \text {for }|\mathbf{t}|>M .
$$

Therefore, when $\lambda<0, \mu>0$, we can deduce that for any $\mathbf{u} \in \mathbf{W}^{p(x)}(\Omega)$ with $\|\mathbf{u}\|_{a}>1$, the following holds

$$
\begin{aligned}
I_{\lambda, \mu}(\mathbf{u}) & \geqslant \frac{1}{p^{+}}\|\mathbf{u}\|_{a}^{p^{-}}-\int_{\Omega} F(x, \mathbf{u}) d x-\mu \int_{\Omega} G(x, \mathbf{u}) d x \\
& \geqslant \frac{1}{p^{+}}\|\mathbf{u}\|_{a}^{p^{-}}+\lambda \epsilon\|\mathbf{u}\|_{a}^{p^{-}}-\mu C\|\mathbf{u}\|_{a}^{\gamma^{+}} .
\end{aligned}
$$

Since $\gamma^{+}<p^{-}, I_{\lambda, \mu}$ is bounded from below and coercive, so (2) is also proved.

Lemma 3.6. Assume that $\left(f_{2}\right.$ and $\left(g_{4}\right.$ hold. Then for any $\lambda\langle 0, \mu\rangle 0$ we have

$$
\inf _{\mathbf{u} \in \mathbf{W}^{p(x)}(\Omega)} I_{\lambda, \mu}(\mathbf{u})<0 .
$$

Proof. Using again assumption $\left(g_{4}\right)$, there exists $\delta>0$ such that

$$
G(x, \mathbf{e}) \geqslant C \mathbf{e}^{\alpha}, \alpha<p^{-}, \text {for all }|\mathbf{e}| \in(0, \delta) .
$$


Choose $\mathbf{v}_{0} \in C_{0}^{\infty}(\Omega)$ such that $0<\mathbf{v}_{0} \leqslant \delta$, and let $\mathbf{u}_{0}=s \mathbf{v}_{0}$. Then by $f_{2}$ and $(3.6)$, for $\lambda<0, \mu>0$, we have

$$
\begin{aligned}
I_{\lambda, \mu}\left(s \mathbf{v}_{0}\right) & \leqslant s^{p^{-}}\left\|\mathbf{v}_{0}\right\|_{a}^{p^{-}}-\lambda \int_{\Omega} F\left(x, s \mathbf{v}_{0}\right) d x-\mu \int_{\Omega} G\left(x, s \mathbf{v}_{0}\right) d x \\
& \leqslant s^{p^{-}}\left\|\mathbf{v}_{0}\right\|_{a}^{p^{-}}+|\lambda| C \int_{\Omega} s^{p(x)}\left|\mathbf{v}_{0}\right|^{p(x)} d x-\mu C \int_{\Omega} s^{\alpha}\left|\mathbf{v}_{0}\right|^{\alpha} d x \\
& \leqslant s^{p^{-}}\left\|\mathbf{v}_{0}\right\|_{a}^{p^{-}}+|\lambda| C s^{p^{-}} \int_{\Omega}\left|\mathbf{v}_{0}\right|^{p(x)} d x-\mu C s^{\alpha} \int_{\Omega}\left|\mathbf{v}_{0}\right|^{\alpha} d x .
\end{aligned}
$$

Since $\alpha<p^{-}$and $s$ is small enough, it follows that $\inf _{\mathbf{u} \in \mathbf{W}^{p(x)}(\Omega)} I_{\lambda, \mu}(\mathbf{u})<0$, which completes the proof.

Proof of Theorem 1.6. By Lemma 3.5, it follows that for any $\lambda<0, \mu>0, I_{\lambda, \mu}$ has a global minimizer $\mathbf{u}_{0}$ to $I_{\lambda, \mu}(\mathbf{u})$ in $\mathbf{W}^{p(x)}(\Omega)$ such that $I_{\lambda, \mu}^{\prime}\left(\mathbf{u}_{0}\right)=0$ (see [40]). Therefore $\mathbf{u}_{0}$ is a weak solution of system (1.5). Moreover, since $I_{\lambda, \mu}(0)=0$ and $I_{\lambda, \mu}\left(\mathbf{u}_{0}\right)<0$ (see Lemma 3.6), u $\neq 0$, i.e. $\mathbf{u}_{0}$ is a nontrivial solution. This completes the proof of Theorem 1.6 .

3.5. Proof of Theorem 1.7. When $\lambda<0, \mu<0$, we argue by contradiction that $\mathbf{u} \in \mathbf{W}^{p(x)}(\Omega) \backslash$ $\{0\}$ is a weak solution of (1.5). Multiplying the first equation of system 1.5 by $\mathbf{u}$, we get

$\int_{\Omega} \nabla \times\left(|\nabla \times \mathbf{u}|^{p(x)-2} \nabla \times \mathbf{u}\right) \cdot \mathbf{u} d x+\int_{\Omega} a(x)|\mathbf{u}|^{p(x)-2} \mathbf{u} \cdot \mathbf{u} d x=\lambda \int_{\Omega} f(x, \mathbf{u}) \cdot \mathbf{u} d x+\mu \int_{\Omega} g(x, \mathbf{u}) \cdot \mathbf{u} d x$.

Using the boundary conditions in 1.5 and integrating by parts, we get

$$
\int_{\Omega}|\nabla \times \mathbf{u}|^{p(x)} d x+\int_{\Omega} a(x)|\mathbf{u}|^{p(x)} d x=\lambda \int_{\Omega} f(x, \mathbf{u}) \cdot \mathbf{u} d x+\mu \int_{\Omega} g(x, \mathbf{u}) \cdot \mathbf{u} d x,
$$

which contradicts $f_{6}$ and $g_{5}$. This completes the proof of Theorem 1.7 .

Acknowledgments. This research was supported by the Slovenian Research Agency grants P10292, N1-0114, N1-0083, N1-0064, and J1-0831.

\section{REFERENCES}

[1] S. Antontsev, M. Chipot, Y. Xie, Uniquenesss results for Equation of the p(x)-Laplacian type, Adv. Math. Sc. Appl., 17 (1) (2007), 287-304.

[2] T. Adamowicz, P. Hästö, Harnack's inequality and the strong $p(\cdot)$-Laplacian, J. Differential Equations, 250 (2011), 1631-1649.

[3] G. A. Afrouzi, M. Mirzapour, N. T. Chung, Existence and multiplicity of solutions for Kirchhoff type problems involving $p(x)$-biharmonic operators, Z. Anal. Anwend., 33 (2014), 289-303.

[4] S. Antontsev, F. Miranda, L. Santos, A class of electromagnetic p-curl systems: blow-up and finite time extinction, Nonlinear Analysis: Theory, Methods and Applications, 75(9) (2012), 3916-3929.

[5] R. Aboulaich, D. Meskine, A. Souissi, New diffusion models in image processing, Comput. Math. Appl., 56 (2008), 874-882.

[6] S. Antontsev, F. Miranda, L. Santos, Blow-up and finite time extinction for $p(x, t)$-curl systems arising in electromagnetism, J. Math. Anal. Appl., 440 (2016), 300-322.

[7] S. N. Antontsev, J. F. Rodrigues, On stationary thermorheological viscous flows, Ann. Univ. Ferrara Sez. VII Sci. Mat., 52 (1) (2006), 19-36.

[8] S. N. Antontsev, S. I. Shmarev, A model porous medium equation with variable exponent of nonlinearity: Existence, uniqueness and localization properties of solutions, Nonlinear Anal., 60 (2005), 515-545.

[9] A. Bahrouni, D. Repovš, Existence and nonexistence of solutions for $p(x)$-curl systems arising in electromagnetism, Complex Variables and Elliptic Equations, 63 (2018), 292-301.

[10] B. Ge, J. F. Lu, Existence and Multiplicity of Solutions for p(x)-Curl Systems Without the AmbrosettiRabinowitz Condition, Mediterranean Journal of Mathematics, 16(2) (2019), p.45

[11] S. Byun, J. Ok, On $W^{1, q(x)}$-estimates for elliptic equations of $p(x)$-Laplacian type, J. Math. Pures Appl., 106 (3) (2016), 512-545.

[12] N. T. Chung, Existence of solutions for perturbed fourth order elliptic equations with variable exponents, Electron. J. Qual. Theory Differ. Equ., 2018 (96) (2018), 1-19.

[13] N. T. Chung, Some remarks on a class of $p(x)$-Laplacian Robin eigenvalue problems, Mediterr. J. Math., 15(4) (2018), p.147 
[14] N. T. Chung, Multiple solutions for a $p(x)$-Kirchhoff-type equation with sign-changing nonlinearities, Complex Var. Elliptic Equ., 58(12) (2013), 1637-1646.

[15] J. Chabrowski, Y. Fu, Existence of solutions for $p(x)$-Laplacian problems on a bounded domain, J. Math. Anal. Appl., 306 (2005), 604-618.

[16] Y. Chen, S. Levine, M. Rao, Variable exponent, linear growth functionals in image restoration, SIAM J. Appl. Math., 66(4) (2006), 1383-1406.

[17] D. E. Edmunds, J. Lang, A. Nekvinda, On $L^{p(x)}$ norms, Proc. Roy. Soc. London Ser. A, 455 (1999), 219-225.

[18] X. L. Fan, D. Zhao, On the spaces $L^{p(x)}$ and $W^{m, p(x)}$, J. Math. Anal. Appl., 263 (2001), 424-446.

[19] Y. Q. Fu, X. Zhang, Multiple solutions for a class of $p(x)$-Laplacian equations in $\mathbb{R}^{N}$ involving the critical exponent, Proc. R. Soc. A, 466 (2010), 1667-1686.

[20] X. L. Fan, S. G. Deng, Remarks on Ricceri's variational principle and applications to the $p(x)$-Laplacian equations, Nonlinear Anal., 67 (2007), 3064-3075.

[21] X. L. Fan, D. Zhao, Nodal solutions of p(x)-Laplacian equations, Nonlinear Anal., 67 (2007), 2859-2868.

$[22]$ B. Ge, L. L. Liu, Infinitely many solutions for differential inclusion problems in $\mathbb{R}^{N}$ involving the $p(x)$-Laplacian, Z. Angew. Math. Phys., 67 (1) (2016), p.16

[23] M. K. Hamdani, A. Harrabi, F. Mtiri, and D. D. Repovš, Existence and multiplicity results for a new p(x)Kirchhoff problem. Nonlinear Analysis 190 (2020): 111598.

[24] M. K. Hamdani, Multiple solutions for Grushin operator without odd nonlinearity. Asian-European J. Math., (2019), doi:10.1142/S1793557120501314

[25] M. K. Hamdani, On nonlocal asymmetric Kirchhoff problem, Asian-European J. Math., (2019), doi: 10.1142/S1793557120300018

[26] M. K. Hamdani, A. Harrabi, High-order Kirchhoff problems in bounded and unbounded domains, (arXiv:1807.11040v3 [math.AP]), 5 Aug 2019.

[27] M. K. Hamdani, A. Harrabi, $L^{\infty}$-norm Estimates of Weak Solutions via their Morse indices for the $m$-Laplacian Problems. Results in Mathematics, 74(1) (2019), p. 69

[28] A. Harrabi, M. K. Hamdani, A. Selmi, Existence results of the zero mass polyharmonic system, Complex Variables and Elliptic Equations (2019), doi:10.1080/17476933.2019.1679794

[29] Y. Hong-Ming, Regularity of weak solution to a p-curl-system, Differential and Integral Equations, 19(4) (2006), 361-368.

[30] O. Kováčik, J. Rákosník; On spaces $L^{p(x)}$ and $W^{k, p(x)}$, Czechoslovak Math. J., 41 (1991), 592-618.

[31] F. Li, Z. Li, L. Pi, Variable exponent functionals in image restoration, Appl. Math. Comput., 216 (3) (2010), 870-882.

[32] M. Mihăsilescu, V. D. Rădulescu, A multiplicity result for a nonlinear degenerate problem arising in the theory of electrorheological fluids, Proc. R. Soc. A 462 (2006), 2625-2641.

[33] M. Mihăilescu, V. D. Rădulescu, On a nonhomogeneous quasilinear eigenvalue problem in Sobolev spaces with variable exponent, Proc. Amer. Math. Soc., 135 (9) (2007), 2929-2937.

[34] K. R. Rajagopal, M. Rüžička, On the modeling of electrorheological materials, Mech. Research Comm., 23 (1996) 401-407.

[35] K. R. Rajagopal, M. Rüžička, Mathematical modeling of electrorheological materials, Cont. Mech. and Thermodynamics, 13 (2001), 59-78.

[36] D. D. Repovš, Stationary waves of Schrödinger-type equations with variable exponent, Anal. Appl. (Singap.), 13 (2015), 645-661.

[37] V. D. Rădulescu, D. D. Repovš, Partial differential equations with variable exponents: variational methods and qualitative analysis, CRC Press, Boca Raton, 2015.

[38] M. Rüžička, Electro-Rheological Fluids: Modeling and Mathematical Theory, Springer-Verlag Berlin, 2000.

[39] M. Q. Xiang, F. L. Wang, B. L. Zhang, Existence and multiplicity of solutions for $p(x)$-curl systems arising in electromagnetism, Journal of Mathematical Analysis and Applications, 448 (2017), 1600-1617.

[40] M. Willem, Minimax Theorems, Birkhäuser, Boston, 1996.

(M.K. Hamdani) Faculty of Science, University of Sfax \& Military School of Aeronautical Specialities, SFAX, Tunisia.

E-mail address: hamdanikarim42@gmail.com

(D.D. Repovš) Faculty of Education and Faculty of Mathematics and Physics, University of Ljubljana \& Institute of Mathematics, Physics and Mechanics, Ljubljana, Slovenia

E-mail address: dusan.repovs@guest.arnes.si 